\title{
Distribution of carnitine and acylcarnitine in the hamster epididymis and in epididymal spermatozoa during maturation
}

\author{
E. R. Casillas, Paula Villalobos and Rita Gonzales \\ Department of Chemistry, New Mexico State University, Las Cruces, New Mexico 88003, U.S.A.
}

\begin{abstract}
Summary. The highest levels of carnitine and acylcarnitine were found in the cauda epididymidis, and spermatozoa from the cauda contained greater amounts of total carnitine (free carnitine plus acylcarnitine) than those removed from the corpus or caput epididymidis. Spermatozoa from the distal cauda contained significantly greater amounts of both free and total carnitine than those removed from the proximal cauda epididymidis. The acylcarnitine: carnitine ratio was 1.7 and 0.37 in caput and cauda spermatozoa, respectively and 1.7 and 1.3 in caput and cauda fluid, respectively. It is suggested that the accumulation of carnitine is involved in sperm maturation and that acylcarnitine serves as an energy substrate for epididymal spermatozoa.
\end{abstract}

\section{Introduction}

The observation that carnitine is present in high concentrations in the rat epididymis was first made by Marquis \& Fritz (1965) and similar observations have been made for rats and other mammals (Casillas, 1972; Brooks, 1980, for review). Carnitine accumulates in bovine spermatozoa as they mature in the epididymis (Casillas, 1973) and there is a close correlation between the carnitine content of rabbit epididymal spermatozoa and their fertility (Casillas \& Chaipayungpan, 1979). Rabbit spermatozoa from epididymal tubules maintained in vitro accumulate carnitine under conditions similar to those during which they become fertile (Casillas \& Chaipayungpan, 1982).

The function of carnitine in spermatozoa and in epididymal fluids has not been proven but several hypotheses have been presented. With regard to intracellular carnitine, our studies with tissue from the cauda epididymidis of bulls showed that carnitine is rapidly acetylated when the spermatozoa are incubated with physiological substrates (Casillas \& Erickson, 1975). Based on these observations, we proposed that the carnitine:acetylcarnitine transferase system serves to buffer against changes in the acetylation state of coenzyme $A$. The result of this effect would be to maximize energy-producing pathways. The effect of substrates on the acetylation state of sperm carnitine has also been demonstrated by Milkowski, Babcock \& Lardy (1976), and Van Dop, Hutson \& Lardy (1977) who have emphasized the role of the acetylcarnitine pool to serve as a readily accessible energy store which can be used when there is a great demand for energy.

With regard to the function of carnitine in epididymal plasma, Hamilton \& Olson (1976) have proposed that the compound serves to depress the respiration of spermatozoa within the epididymis. In addition, Storey \& Keyhani (1974) have suggested that extracellular acetylcarnitine serves as an energy substrate for spermatozoa as they pass through the epididymis. This suggestion is supported by the observation that, in some species, e.g. rabbit, the epididymis contains high amounts of acetylcarnitine (Casillas \& Chaipayungpan, 1979). 
We have now examined the distribution of carnitine and acylcarnitine in hamster epididymal spermatozoa and tissue because the 'fertility profile' of spermatozoa removed from various regions of the epididymis is known (Horan \& Bedford, 1972) and is different from that of the rabbit (Bedford, 1966; Orgebin-Crist, 1967), with significant numbers of fertile spermatozoa first appearing in the proximal cauda epididymidis. We hoped to correlate the carnitine content of epididymal spermatozoa with their fertility and to measure the acylation state of carnitine in epididymal tissue.

\section{Materials and Methods}

Preparation of epididymal and sperm extracts. Epididymides from mature Syrian hamsters were removed and carefully trimmed of adipose and connective tissue and of superficial blood vessels. The organs were divided into caput, corpus and cauda sections and the cauda epididymidis was sometimes further divided into 4 sections (Text-fig. 1). Equivalent sections from epididymides and animals were pooled and then lacerated with a sharp scapel, incubated at $37^{\circ} \mathrm{C}$ and gently stirred in Dulbecco's medium for $30 \mathrm{~min}$. The spermatozoa which were released into the medium were isolated according to the methods of Harrison (1976): $3 \mathrm{ml}$ medium were layered over a $10 \mathrm{ml}$ solution containing $7.5 \%$ Ficoll and $7.5 \%$ sucrose in a conical centrifuge tube and centrifuged for 10 min at $1200 \mathrm{~g}$ at ambient temperature. Spermatozoa present in the pellet were washed once more through Ficoll-sucrose before being analysed for carnitine content. Results from preliminary experiments showed that the carnitine and acylcarnitine content of spermatozoa from various regions of the epididymis remained constant upon further washing, an indication that the washing procedure removed extracellular but not intracellular carnitine. Microscopic examination of sperm preparations from all regions showed them to be at least $95 \%$ pure. Sperm counts were performed by conventional haemocytometer techniques and purity was estimated by comparing the number of spermatozoa obtained during cell-counting procedures to the total number of cells observed in the same field. The original supernatant solution from the incubation was combined with the supernatant solutions obtained from the washes and this fraction (referred to as epididymal fluid) was analysed for carnitine and acylcarnitine content.

Analysis of carnitine and acylcarnitine. Extracts for the analyses of carnitine and short-chain acylcarnitine were prepared according to the procedure described by Pearson \& Tubbs (1964) as modified by Casillas (1972). In brief, spermatozoa suspended in $0.5 \mathrm{M}-\mathrm{HClO}_{4}$ were disrupted further by sonic irradiation. The epididymal fluid solutions were acidified by the addition of sufficient $5 \mathrm{M}-\mathrm{HClO}_{4}$ to yield a final concentration of $0.5 \mathrm{M}$. In both cases, acid-insoluble material was removed by centrifugation and the acid-soluble fraction was neutralized with $\mathrm{KOH}$. The $\mathrm{KClO}_{4}$ precipitate was removed by another centrifugation and the carnitine present in the clear neutral extracts was determined by the method described by Cederblad \& Lindstedt (1972) with modifications suggested by McGarry \& Foster (1976). The limit of sensitivity was 5 pmol and the relative deviation of 5 duplicate analyses was $2-10 \%$. In 3 experiments, the recovery of carnitine added to perchloric acid extracts of spermatozoa was $100 \pm 10 \%$. Acylcarnitine, present in the $\mathrm{HClO}_{4}$-soluble fraction, was estimated by assaying for carnitine liberated by the hydrolytic procedure described by Pearson \& Tubbs (1964).

Materials. Carnitine acetyltransferase from pigeon breast muscles ( 98 units/mg protein) was obtained from Sigma Chemical Co., St Louis, MO; L-carnitine chloride was from Supelco Inc., Bellefonte, PA; [acetyl- ${ }^{3} \mathrm{H}$ ]acetyl Coenzyme A (sp. act. $1.97 \mathrm{Ci} / \mathrm{mmol}$ ) was from New England Nuclear, Boston, MA; Dowex $2 \times 8$ (200-400 mesh) was from Biorad Labs, Richmond, CA; sodium tetrathionate was from ICN Pharmaceuticals, Plainview, NY; Ficoll 400 was from Pharmacia, Uppsala, Sweden. All other reagents were of the best grade available from commercial sources. 


\section{Results}

The carnitine and acylcarnitine content of spermatozoa removed from the caput, corpus and cauda is shown in Text-fig. 1. There was a significant increase in free and total carnitine (the sum of carnitine and acylcarnitine) as the cells passed from the corpus to the cauda. The amount of acylcarnitine was significantly greater in spermatozoa from the caput than in those from the corpus and in all cases constituted a greater percentage of the total carnitine pool than has been observed in other species (see 'Discussion').

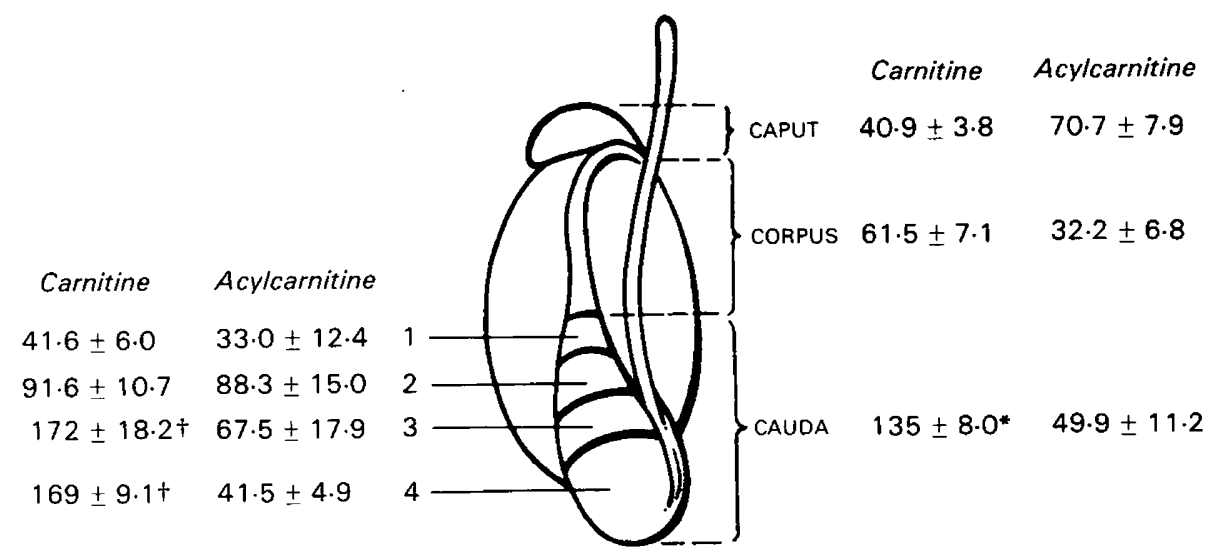

Text-fig. 1. Diagrammatic representation of the hamster testis and epididymis showing the regions used in the study, and the carnitine and acylcarnitine contents ( $\mathrm{nmol} / 10^{8}$ cells) of spermatozoa removed from them. Values are mean \pm s.e.m. for 6 separate experiments on 8-12 epididymides/experiment for caput, corpus and cauda sections and for 5 separate experiments on 4 caudae/experiment for the cauda regions. ${ }^{*} P<0.01$ compared with values for caput and cauda (Student's $t$ test). $† P<0.01$ compared with values for regions 1 and 2 (Student's $t$ test).

The carnitine and acylcarnitine contents of spermatozoa removed from various regions of the cauda epididymidis are shown in Text-fig. 1. The largest and most significant increase in free and total carnitine occurred as the cells passed from region 2 to region 3 of the cauda. In general, the acylcarnitine content was more constant than that of free carnitine but the percentage of the total carnitine represented by acylcarnitine dropped in regions 3 and 4 . The acylcarnitine content as a percentage of the total was $44,49,28$ and $20 \%$ for regions $1,2,3$ and 4 , respectively.

The distributions of the free carnitine and acylcarnitine in fluid from various segments of the epididymis are shown in Table 1 . The data represent the amount of non-sperm carnitine and acylcarnitine that was washed out of the segments by lacerating them and incubating them in a saltbuffer. Results from preliminary experiments showed that this procedure extracted at least $90 \%$ of the total carnitine and acylcarnitine present in the segments when total carnitine was measured by extracting the segments with dilute perchloric acid (Pearson \& Tubbs, 1964). The fact that the compounds were easily washed from incised tissue suggests that they were derived from epididymal

Table 1. Distribution of carnitine and acylcarnitine in hamster epididymal fluid

\begin{tabular}{lccccc}
\hline & \multicolumn{2}{c}{ Carnitine } & & \multicolumn{2}{c}{ Acylcarnitine } \\
\cline { 2 - 3 } \cline { 5 - 6 } Region & nmol/g wet wt & nmol/epididymis & & nmol/g wet & nmol/epididymis \\
\hline Caput & $19 \cdot 3 \pm 2 \cdot 2$ & $2 \cdot 4 \pm 0 \cdot 28$ & & $33 \cdot 1 \pm 4 \cdot 2$ & $4 \cdot 16 \pm 0 \cdot 54$ \\
Corpus & $14 \cdot 9 \pm 0 \cdot 6$ & $1 \cdot 1 \pm 0 \cdot 04$ & & $7 \cdot 3 \pm 0 \cdot 24$ & $0 \cdot 55 \pm 0.02$ \\
Cauda & $357 \pm 46$ & $117 \pm 14$ & & $480 \pm 63$ & $156 \pm 20$ \\
\hline
\end{tabular}

Values are mean \pm s.e.m. from 4 different experiments with 12 epididymides per experiment. 
plasma rather than from the tissue itself. Fluid from the cauda contained far greater amounts of free carnitine and acylcarnitine than did fluid from the caput and corpus epididymidis.

\section{Discussion}

The results presented here extend our previous observations that carnitine accumulates in bovine (Casillas, 1973) and rabbit (Casillas \& Chaipayungpan, 1979) spermatozoa as they progress through the epididymis. Furthermore, as with rabbits, we have shown that increases in the carnitine content of spermatozoa removed from various regions of the hamster epididymis are closely correlated with the development of fertility of these cells as reported by Horan \& Bedford (1972). For example, only a small percentage of cells from the proximal cauda (region 1) were mature and the cells from region 2 fertilized only $15 \%$ of the eggs recovered after insemination of the test animals. In contrast, cells from regions 3 and 4 , those that contain the highest carnitine content (present study), fertilized 89 and $79 \%$ respectively of the recovered eggs. Our contention that carnitine accumulation is an integral process of sperm maturation is further supported by the observations that although the fertility profile of spermatozoa removed from various regions of the hamster and rabbit epididymis is different, increases in the carnitine content of the cells is closely correlated to the development of fertility. The probable consequence of carnitine accumulation during maturation is that mature cells have an increased capacity for energy metabolism which is used to sustain highly motile cells after ejaculation (Carter, Stratman, Hutson \& Lardy, 1980).

Our results on the distribution of carnitine in fluids from various regions of the epididymis show the same pattern as has been found in a variety of species (see review by Brooks, 1980). That is, the concentration of carnitine is much greater in the cauda than in other sections of the epididymis.

A finding that is unusual in the present study is that the acylation state of carnitine in the fluids and spermatozoa from the hamster epididymis is greater than that which is reported for other species. Although in the present study we did not establish definitively that the acylcarnitine was indeed acetylcarnitine, in our previous studies with bulls and rabbits we showed that essentially all of the carnitine liberated by mild base hydrolysis of the perchloric-acid extracts was due to the hydrolysis of acetylcarnitine. If this is the case in the present study, then acetylcarnitine makes up 63,33 and $57 \%$ of the total perchloric acid-soluble carnitine in fluid from the caput, corpus and cauda epididymidis, respectively. In other species the values are about $30 \%$ in the rabbit caput and $40 \%$ in the corpus and cauda (Casillas \& Chaipayungpan, 1979), about $25 \%$ in the bovine caput and $6 \%$ in the cauda (Casillas, 1972), and about $10 \%$ in both the caput and cauda of rats (Brooks, Hamilton \& Mallek, 1974). The high levels of acylcarnitine in the epididymis suggest that it might be used as an energy source by spermatozoa as they progress through the epididymis. This idea is further supported by comparing data in Text-fig. 1 and Table 1. The acylcarnitine:carnitine ratio changes from 1.7 in caput spermatozoa to 0.37 in cauda spermatozoa, while the same ratio changes from 1.7 in caput fluid to 1.3 in cauda fluid. These changes in the acylcarnitine:carnitine ratio during epididymal transit might reflect an increase in the metabolic activity of spermatozoa as they mature.

The origin of epididymal acetylcarnitine or perhaps other short-chain acylcarnitines (Golan et al., 1983) is unknown. Golan et al.(1983) suggest that acetylcarnitine in the epididymal lumen is produced by spermatozoa and hence is a by-product of sperm metabolism. This seems unlikely since many attempts have been made to identify energy substrates for spermatozoa in epididymal fluids and have not yielded conclusive results. In reviewing this subject, Brooks (1979) has suggested that, of the several compounds available to spermatozoa in epididymal plasma, lactate and glycerol might be present in sufficient quantities to meet the energy requirements of the cells. However, his calculations assume the complete oxidation of lactate to $\mathrm{CO}_{2}$. Only a fraction of the required energy would result if lactate were converted to acetyl-Co $\mathrm{A}$ and then acetate units were lost to the epididymal plasma as acetylcarnitine. The same argument would, of course, apply in the 
case of glycerol metabolism. It seems more likely that acetylcarnitine originates from another source, perhaps the epididymal epithelium, and is used by spermatozoa as an energy substrate.

This work was supported by grants from the National Institutes of Health (HD 10664, RR 08136).

\section{References}

Bedford, J.M. (1966) Development of the fertilizing ability of spermatozoa in the epididymis of the rabbit. J. exp. Zool. 163, 319-339.

Brooks, D.E. (1979) Biochemical environment of sperm maturation. In The Spermatozoon, Maturation, Motility, Surface Properties, and Comparative Aspects, pp. 23-34. Eds D. W. Fawcett \& J. M. Bedford. Urban \& Schwarzenburg, Baltimore.

Brooks, D.E. (1980) Carnitine in the male reproductive tract and its relation to the metabolism of the epididymis and spermatozoa. In Carnitine Biosynthesis, Metabolism, and Functions, pp. 219-235. Eds R. A. Frenkel \& J. D. McGarry. Academic Press, New York.

Brooks, D.E., Hamilton, D.W. \& Mallek, A.H. (1974) Carnitine and glycerylphosphorylcholine in the reproductive tract of the male rat. J. Reprod. Fert. 36, 141-160.

Carter, A.L., Stratman, F.W., Hutson, S.M. \& Lardy, H.A. (1980) The role of carnitine and its esters in sperm metabolism. In Carnitine Biosynthesis, Metabolism, and Functions, pp. 251-269. Eds R. A. Frenkel \& J. D. McGarry. Academic Press, New York.

Casillas, E.R. (1972) The distribution of carnitine in male reproductive tissues and its effect on palmitate oxidation by spermatozoal particles. Biochim. Biophys. Acta 280, 545-551.

Casillas, E.R. (1973) Accumulation of carnitine by bovine spermatozoa during maturation in the epididymis. J. biol. Chem. 248, 8227-8232.

Casillas, E.R. \& Chaipayungpan, S. (1979) The distribution of carnitine and acetylcarnitine in the rabbit epididymis and the carnitine content of rabbit spermatozoa during maturation. J. Reprod. Fert. 56, 439-444.

Casillas, E.R. \& Chaipayungpan, S. (1982) Carnitine content of rabbit epididymal spermatozoa in organ culture. J. Reprod. Fert. 65, 247-251.

Casillas, E.R. \& Erickson, B.J. (1975) The role of carnitine in spermatozoan metabolism: substrateinduced elevations in the acetylation state of carnitine and coenzyme- $A$ in bovine and monkey spermatozoa. Biol. Reprod. 12, 275-283.
Cederblad, B. \& Lindstedt, S. (1972) A method for the determination of carnitine in the picomole range. Clin. chim. Acta 37, 235-243.

Golan, R., Soffer, Y., Katz, S., Weissenberg, R., Wasserzug, O. \& Lewin, L.M. (1983) Carnitine and short-chain acylcarnitines in the lumen of the human male reproductive tract. Int. J. Androl. 6, 349-357.

Hamilton, D.W. \& Olson, G.E. (1976) Effects of carnitine on oxygen uptake and utilisation of $\left[\mathrm{U}-{ }^{14} \mathrm{C}\right]$ palmitate by ejaculated bull spermatozoa. J. Reprod. Fert. 46, 195-202.

Harrison, R.A.P. (1976) A highly efficient method for washing mammalian spermatozoa. J. Reprod. Fert. 48, 347-353.

Horan, A.H. \& Bedford, J.M. (1972) Development of the fertilizing ability of spermatozoa in the epididymis of the Syrian hamster. J. Reprod. Fert. 30, 417-423.

Marquis, N.R. \& Fritz, I.B. (1965) The distribution of carnitine, acetylcarnitine, and carnitine acetyltransferase in rat tissues. J. biol. Chem. 240, 2193-2196.

McGarry, J.D. \& Foster, D.W. (1976) An improved and simplified radioisotopic assay for the determination of free and esterified carnitine. J. Lipid Res. 17, 277280.

Milkowski, A.L., Babcock, D.F. \& Lardy, H.A. (1976) Activation of bovine epididymal sperm respiration by caffeine. Its transient nature and relationship to the utilization of acetylcarnitine. Archs Biochem. Biophys. 176, 250-256.

Orgebin-Crist, M.C. (1967) Maturation of spermatozoa in the rabbit epididymis; fertilizing ability and embryonic mortality in does inseminated with epididymal spermatozoa. Annls Biol. anim. Biochim. Biophys. 7, 373-389.

Pearson, P.J. \& Tubbs, P.K. (1964) Tissue levels of acidinsoluble carnitine in rat heart. Biochim. Biophys. Acta 84, 772-773.

Storey, B.T. \& Keyhani, E. (1974) Energy metabolism of spermatozoa. II. Comparison of pyruvate and fatty acid oxidation by mitochondria of rabbit epididymal spermatozoa. Fert. Steril. 25, 857-864.

Van Dop, C., Hutson, S.M. \& Lardy, H.A. (1977) Pyruvate metabolism in bovine epididymal spermatozoa. J. biol. Chem. 252, 1303-1308. 